\title{
Impact of diabetes mellitus on long term survival after acute myocardial infarction in patients with single vessel disease
}

\author{
M Ishihara, H Sato, T Kawagoe, Y Shimatani, S Kurisu, K Nishioka, Y Kouno, \\ T Umemura, S Nakamura
}

\begin{abstract}
Objective-To assess the influence of diabetes on long term prognosis after reperfusion treatment and its interaction with multivessel disease.

Design-A retrospective observational study.

Setting-Hiroshima City Hospital.

Patients-1660 consecutive patients with acute myocardial infarction who underwent coronary angiography within 24 hours after the onset of chest pain.

Main outcome measures-Influence of diabetes on 10 year survival after infarction was assessed using the generalised Wilcoxon test and Cox's proportional hazards regression. Follow up was completed in 1622 patients (98\%).

Results-Diabetic patients had more multivessel disease than non-diabetic patients (53\% $v$ $34 \%, \mathrm{p}<0.001)$. When only patients with single vessel disease were compared, diabetes was associated with a reduced 10 year survival after infarction $(p=0.002)$. On the other hand, in patients with multivessel disease there was no significant difference in survival between diabetic and non-diabetic patients $(\mathrm{p}=0.70)$. Multivariate analysis also showed that diabetes was an independent risk factor related to 10 year mortality after infarction in patients with single vessel disease (odds ratio (OR) 1.81, 95\% confidence interval (CI) 1.27 to $2.54 ; \mathrm{p}=0.001$ ) and not in patients with multivessel disease (OR $1.17,95 \%$ CI 0.85 to $1.60 ; \mathrm{p}=0.34$ ).

Conclusions-Diabetes is an independent predictor of long term mortality after infarction in patients with single vessel disease. However, in the presence of multivessel disease, prognosis after infarction is impaired regardless of diabetes, and the influence of diabetes is less obvious.

(Heart 2001;86:133-138)
\end{abstract}

Keywords: myocardial infarction; diabetes; coronary angiography

Diabetes mellitus is a risk factor for the development of cardiovascular disease. ${ }^{1}$ A quarter of patients with acute myocardial infarction have diabetes. It was reported in the prereperfusion era that patients with diabetes had a worse prognosis after myocardial infarction than non-diabetic patients. ${ }^{2-5}$ Although recent studies have also shown that diabetes impairs survival after reperfusion treatment for acute myocardial infarction, most of these reports are limited to a short term follow up period and there are few reports of long term outcome with modern management. ${ }^{6-8}$ In the present study, we assessed the influence of diabetes on 10 year survival after myocardial infarction in the reperfusion era.

Diabetic patients have a worse risk profile than non-diabetic patients. Although several studies have shown that diabetes is an independent predictor of mortality after myocardial infarction, most of these analyses did not include angiographic findings. Diabetic patients have more extensive coronary artery disease, and multivessel disease is a strong predictor of mortality after myocardial infarction. ${ }^{9-11}$ A previous study reported that the relative risk of mortality after myocardial infarction in diabetes was greatest among patients with the lowest clinical risk and least among those with the highest risk, but the interaction between diabetes and multivessel disease has not yet been clarified. ${ }^{12}$ It is important to determine whether diabetes has more impact on survival after myocardial infarction in patients with single vessel disease than in multivessel disease, as physicians generally pay less attention to the former than to the latter. In order to explore this issue further, we reviewed the acute angiographic findings in our patients with myocardial infarction.

\section{Methods}

STUDY PATIENTS

Between January 1981 and December 1999, 1660 consecutive Japanese patients with acute myocardial infarction underwent coronary angiography within 24 hours after the onset of chest pain in Hiroshima City Hospital. We chose a time window of 24 hours because recent studies have reported that reperfusion initiated 6-24 hours after the onset of chest pain prevents extension of the infarct and improves the outcome. ${ }^{13}$ Acute myocardial infarction was diagnosed by chest pain consistent with ongoing myocardial ischaemia persisting for longer than 30 minutes, with concomitant ECG changes. We did not determine whether chest pain was still present at the time of study entry. ${ }^{14}$ Serum creatine kinase was measured every three hours for at least 24 hours, and the peak creatine kinase value had to be more than twice the normal upper limit. 
Table 1 Characteristics of patients with single vessel disease

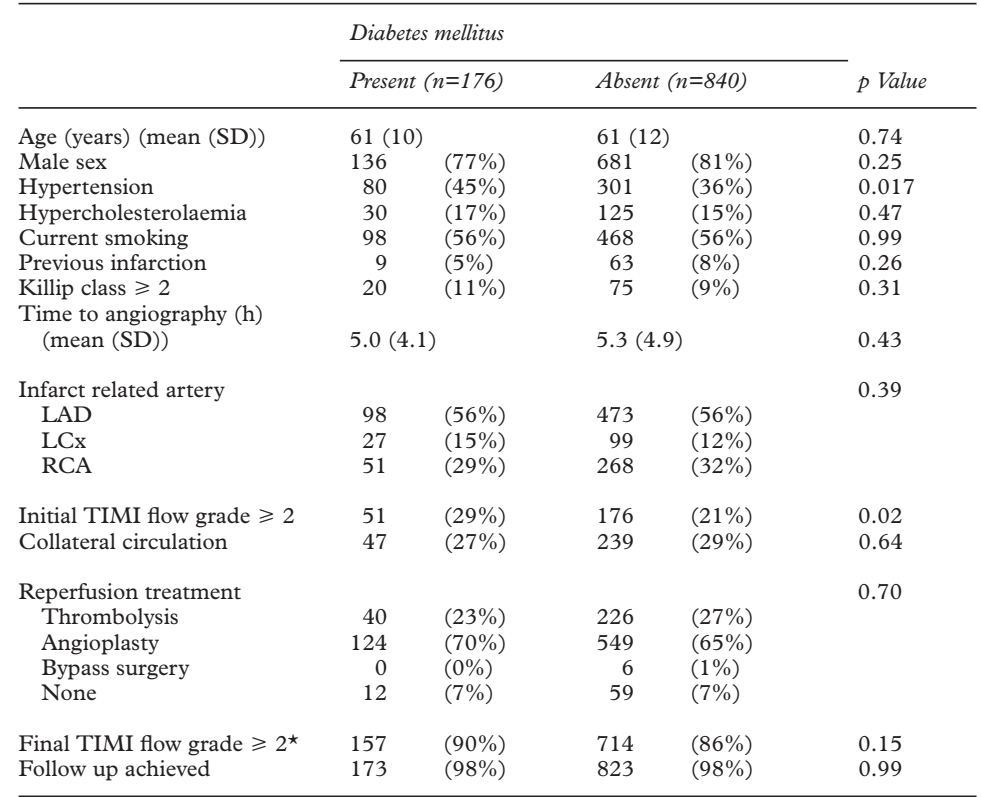

${ }^{\star}$ Data were not obtained in patients undergoing bypass surgery.

$\mathrm{LAD}$, left anterior descending coronary artery; LCx, left circumflex coronary artery; LMT, left main trunk, RCA, right coronary artery; TIMI, thrombolysis in myocardial infarction trial.

\section{CARDIAC CATHETERISATION}

Emergency cardiac catheterisation was performed through the right femoral artery after heparin administration. Selective coronary angiography was performed in multiple projections before initiating reperfusion treatment. After diagnostic angiography, reperfusion treatment was undertaken if indicated. The allocation to thrombolysis or angioplasty was not randomised and was based on the physician's decision. In general, thrombolytic treatment was given without adjunctive angioplasty during the first four years. During the last 15 years, angioplasty was performed for severe stenosis after thrombolysis, after failed thrombolysis, or where thrombolytic treatment was not given for any reason. ${ }^{15}$

\section{ANGIOGRAPHIC ANALYSIS}

All coronary angiograms were reviewed by two angiographers who had no knowledge of the clinical variables. The perfusion status of the infarct related coronary artery was determined in accordance with the TIMI (thrombolysis in myocardial infarction) study classification. ${ }^{16}$ An occluded artery was defined as TIMI flow grade $\leqslant 1$; reperfusion was defined as TIMI flow grade $\geqslant 2$. Initial TIMI flow grade was assessed before the initiation of reperfusion treatment and final TIMI flow grade on the final shot of the angiogram. Multivessel coronary disease was defined as $\geqslant 75 \%$ stenosis in one or more vessels remote from the infarct related artery. Left main coronary disease was considered to be at least two vessel involvement. The extent of collateral circulation was assessed on the pretreatment angiogram and classified by the method described by Rentrop and colleagues. ${ }^{17}$ Collateral circulation was considered to be present if the grade was $\geqslant 2$.
DATA ANALYSIS

During the hospital admission data were collected on whether the patients had a previous or current diagnosis of diabetes mellitus. In-hospital events were defined as death, shock, congestive heart failure, and reinfarction. Follow up was achieved for up to 10 years in 1622 patients $(98 \%)$ by reviewing clinical records or by means of a form mailed to the patients or their families. Mean (SD) follow up duration was 5.2 (3.6) years.

Statistical analysis was performed using $\chi^{2}$ and $t$ tests. Kaplan-Meier estimates were used to construct a long term survival curve. Differences in 10 year survival were assessed using the generalised Wilcoxon test. The Cox proportional hazards regression was used to identify independent predictors of 10 year survival, simultaneously assessing variables including age, sex, hypertension, hypercholesterolaemia, current smoking, previous infarction, Killip class at the time of hospital admission, time to angiography, initial patency, collateral circulation, multivessel disease, and final reperfusion. Differences were considered significant at a probability value of $\mathrm{p}<0.05$.

\section{Results}

CLINICAL AND ANGIOGRAPHIC CHARACTERISTICS

There were 378 patients with diabetes $(27 \%)$ : 178 of these $(47 \%)$ were treated with diet alone, $172(46 \%)$ with oral hypoglycaemic drugs, and $28(7 \%)$ with insulin. Diabetes was more common in women than men $(25 \% v$ $19 \%, \mathrm{p}=0.006)$, and was associated with hypertension ( $46 \% v 38 \%, \mathrm{p}=0.003)$, Killip class $\geqslant 2(17 \% v 12 \%, \mathrm{p}=0.015)$, and multivessel disease $(53 \%$ v 34\%, $\mathrm{p}<0.001)$.

When the 1016 patients with single vessel disease were compared, the only significant difference in clinical and angiographic variables between diabetic and non-diabetic patients was that there was more hypertension in the diabetic group and a greater likelihood that the infarct related artery was initially patent (table 1). Among the 642 patients with multivessel disease, there was no significant difference in these variables, expect that diabetic patients were more likely to be female (table 2).

\section{IN-HOSPITAL OUTCOME}

During the hospital admission, 148 patients died $(8.9 \%)$. In-hospital mortality was significantly greater in diabetic than in non-diabetic patients $(12.2 \% v 8.0 \%, p=0.012)$. There was no major difference in the cause of death in diabetic compared with non-diabetic patients. The in-hospital event rate was higher in diabetic than non-diabetic patients $(25.4 \% v$ $17.9 \%, p=0.001)$. Peak creatine kinase was obtained in 1496 patients (90\%). The mean (SD) value was 2679 (2077) IU/1 in diabetic patients and 2655 (2228) IU/1 in non-diabetic patients $(\mathrm{p}=0.86)$.

When the 1016 patients with single vessel disease were compared, in-hospital mortality of diabetic patients was $6.3 \%$ and of non-diabetic patients, $3.9 \%(p=0.17)$. Diabetes was associated with more in-hospital events $(18.8 \% v$ 
Table 2 Characteristics of patients with multivessel disease

\begin{tabular}{|c|c|c|c|c|c|}
\hline \multirow[b]{3}{*}{ Age (years) (mean (SD)) } & \multicolumn{4}{|c|}{ Diabetes mellitus } & \multirow{3}{*}{$\frac{p \text { Value }}{0.63}$} \\
\hline & \multicolumn{2}{|c|}{ Present $(n=202)$} & \multicolumn{2}{|c|}{ Absent $(n=442)$} & \\
\hline & 64 & (10) & 63 & (11) & \\
\hline Male sex & 146 & $(72 \%)$ & 358 & $(81 \%)$ & 0.01 \\
\hline Hypertension & 94 & $(47 \%)$ & 183 & $(42 \%)$ & 0.19 \\
\hline Hypercholesterolaemia & 32 & $(16 \%)$ & 64 & $(14 \%)$ & 0.65 \\
\hline Current smoking & 98 & $(49 \%)$ & 216 & $(49 \%)$ & 0.93 \\
\hline Previous infarction & 42 & $(21 \%)$ & 99 & $(22 \%)$ & 0.65 \\
\hline Killip class $\geqslant 2$ & 44 & $(22 \%)$ & 80 & $(18 \%)$ & 0.27 \\
\hline $\begin{array}{l}\text { Time to angiography (h) } \\
(\text { mean }(\mathrm{SD}))\end{array}$ & 6.1 & $(5.5)$ & 5.6 & $(5.1)$ & 0.27 \\
\hline Infarct related artery & & & & & 0.21 \\
\hline LAD & 82 & $(41 \%)$ & 185 & $(42 \%)$ & \\
\hline $\mathrm{LCx}$ & 32 & $(16 \%)$ & 73 & $(17 \%)$ & \\
\hline RCA & 84 & $(42 \%)$ & 158 & $(36 \%)$ & \\
\hline LMT & 4 & $(2 \%)$ & 26 & $(6 \%)$ & \\
\hline Initial TIMI flow grade $\geqslant 2$ & 46 & $(23 \%)$ & 101 & $(23 \%)$ & 0.88 \\
\hline Collateral circulation & 63 & $(31 \%)$ & 146 & $(33 \%)$ & 0.64 \\
\hline Reperfusion treatment & & & & & 0.64 \\
\hline Thrombolysis & 48 & $(24 \%)$ & 168 & $(71 \%)$ & \\
\hline PTCA & 118 & $(58 \%)$ & 124 & $(52 \%)$ & \\
\hline Bypass surgery & 27 & $(13 \%)$ & 23 & $(10 \%)$ & \\
\hline None & 9 & $(4 \%)$ & 19 & $(8 \%)$ & \\
\hline Final TIMI flow grade $\geqslant 2^{\star}$ & 148 & $(85 \%)$ & 309 & $(81 \%)$ & 0.21 \\
\hline Follow up achieved & 197 & $(98 \%)$ & 429 & $(97 \%)$ & 0.94 \\
\hline
\end{tabular}

^Data were not obtained in patients undergoing bypass surgery.

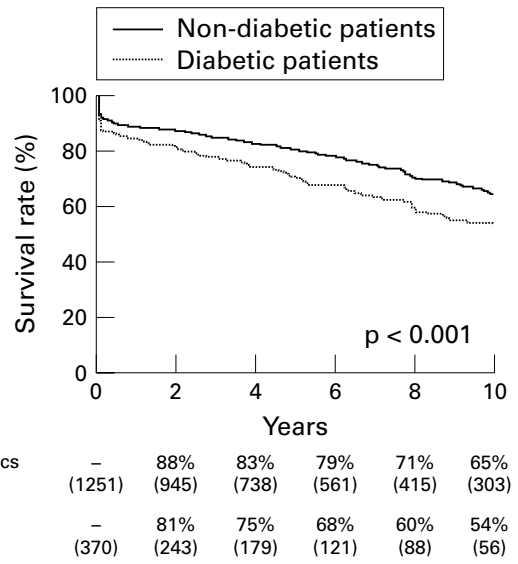

$\begin{array}{lcccccc}\begin{array}{l}\text { Non-diabetics } \\ \text { (n) }\end{array} & - & 88 \% & 83 \% & 79 \% & 71 \% & 65 \% \\ \begin{array}{l}\text { Diabetics } \\ \text { (n) }\end{array} & - & 81 \% & 75 \% & 68 \% & 60 \% & 54 \% \\ & (370) & (243) & (179) & (121) & (88) & (56)\end{array}$

Figure 110 year survival curves for all enrolled patients. Diabetic patients had significantly worse survival after myocardial infarction than non-diabetic patients.

$12.6 \%, p=0.031)$. Among patients with multivessel disease, there was no significant difference in in-hospital mortality $(17.3 \% v 15.6 \%$, $\mathrm{p}=0.58)$ or in the in-hospital event rate $(31.2 \%$ v $27.8 \%, p=0.38)$ between diabetic and non-diabetic patients.

LONG TERM SURVIVAL

During the 10 year follow up period, 434 patients died. One year, five year, and 10 year survival rates were $88 \%, 79 \%$, and $63 \%$, respectively. Survival during 10 years after myocardial infarction was significantly less likely in diabetic than in non-diabetic patients ( $p<0.001$, fig 1). Patients with multivessel disease had a worse 10 year survival than those with single vessel disease $(p<0.001$, fig 2$)$.

When only the patients with single vessel disease were compared, 10 year survival after myocardial infarction was still worse in diabetic than in non-diabetic patients $(\mathrm{p}=0.002$, fig 3$)$. However, among patients with multivessel disease, there was no significant difference in 10

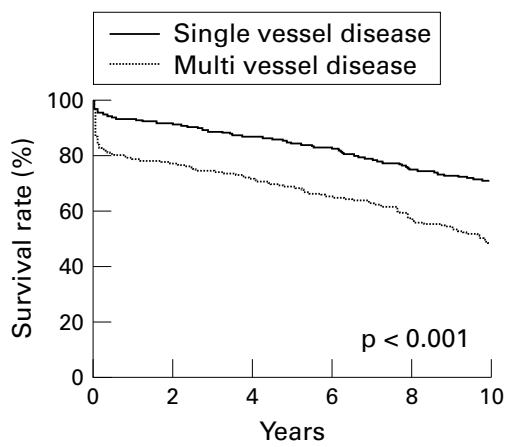

$\begin{array}{lcccccc}\begin{array}{l}\text { Single vessel } \\ \text { (n) }\end{array} & - & 92 \% & 87 \% & 83 \% & 75 \% & 71 \% \\ \begin{array}{l}\text { Multi vessel } \\ \text { (n) }\end{array} & - & 78 \% & 72 \% & 65 \% & 58 \% & 48 \% \\ & (626) & (409) & (290) & (207) & (142) & (95)\end{array}$

Figure 210 year survival curves for all enrolled patients. Patients with multivessel disease had significantly worse survival after myocardial infarction than those with single vessel disease.

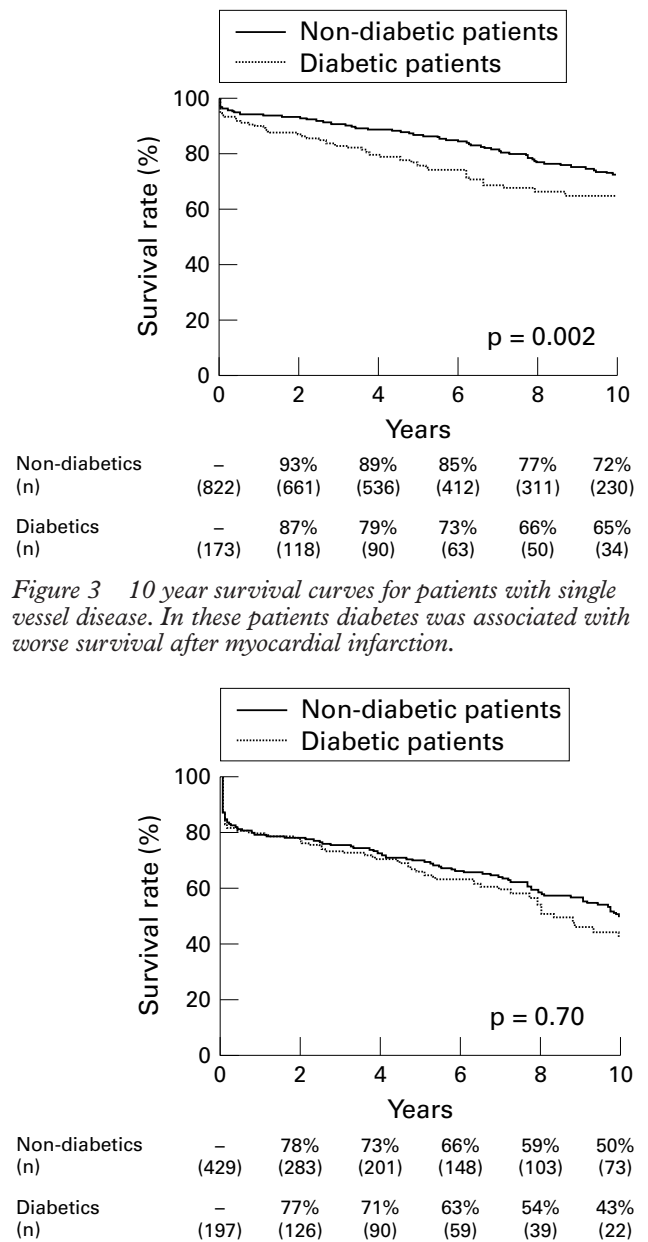

Figure 410 year survival curves for patients with multivessel disease. In patients with multivessel disease, survival after myocardial infarction was poor regardless of diabetes.

year survival after infarction between diabetic and non-diabetic patients ( $\mathrm{p}=0.70$, fig 4 ).

Multivariate analysis was performed to assess predictors of death during the 10 year follow up period, adjusting for age, sex, hypertension, hypercholesterolaemia, current smoking, previous infarction, Killip class, time to angiography, initial patency, collateral circulation, and final reperfusion. This showed that 
Table 3 Multivariate analysis assessing predictors of death during a 10 year follow up in patients with single vessel disease

\begin{tabular}{llc}
\hline & Odds ratio (95\% CI) & p Value \\
\hline Diabetes mellitus & $1.81(1.27$ to 2.54$)$ & 0.001 \\
Age & $1.07(1.05$ to 1.08$)$ & $<0.001$ \\
Male sex & $0.77(0.52$ to 1.12$)$ & 0.17 \\
Hypertension & $1.24(0.93$ to 1.66$)$ & 0.15 \\
Hypercholesterolaemia & $0.75(0.47$ to 1.12$)$ & 0.49 \\
Current smoker & $1.24(0.91$ to 1.69$)$ & 0.18 \\
Previous infarction & $1.21(0.68$ to 2.01$)$ & 0.49 \\
Killip class $\geqslant 2$ & $2.01(1.31$ to 3.00$)$ & 0.002 \\
Time to angiography $\geqslant 6 \mathrm{~h}$ & $1.18(0.87$ to 1.59$)$ & 0.29 \\
Anterior location & $1.29(0.96$ to 1.73$)$ & 0.09 \\
Initial TIMI flow grade $\geqslant 2$ & $1.14(0.79$ to 1.63$)$ & 0.47 \\
Collateral circulation & $1.12(0.80$ to 1.56$)$ & 0.51 \\
Final TIMI flow grade $\geqslant 2$ & $0.52(0.36$ to 0.76$)$ & $<0.001$ \\
\hline CI, confidence interval. & \multicolumn{2}{l}{}
\end{tabular}

Table 4 Multivariate analysis assessing predictors of death during a 10 year follow up in patients with multivessel disease

\begin{tabular}{llc}
\hline & Odds ratio $(95 \%$ CI) & p Value \\
\hline Diabetes mellitus & $1.16(0.85$ to 1.60$)$ & 0.34 \\
Age & $1.06(1.04$ to 1.08$)$ & $<0.001$ \\
Male sex & $1.31(0.91$ to 1.85$)$ & 0.14 \\
Hypertension & $1.19(0.88$ to 1.62$)$ & 0.26 \\
Hypercholesterolaemia & $0.56(0.34$ to 0.89$)$ & 0.34 \\
Current smoker & $1.11(0.81$ to 1.52$)$ & 0.012 \\
Previous infarction & $2.07(1.49$ to 2.84$)$ & $<0.001$ \\
Killip class $\geqslant 2$ & $3.30(2.34$ to 4.57$)$ & $<0.001$ \\
Time to angiography $\geqslant 6 \mathrm{~h}$ & $1.05(0.75$ to 1.44$)$ & 0.80 \\
Anterior location & $1.16(0.86$ to 1.56$)$ & 0.32 \\
Initial TIMI flow grade $\geqslant 2$ & $1.23(0.82$ to 1.81$)$ & 0.31 \\
Collateral circulation & $0.90(0.64$ to 1.24$)$ & 0.52 \\
Final TIMI flow grade $\geqslant 2$ & $0.53(0.38$ to 0.75$)$ & $<0.001$ \\
\hline
\end{tabular}

diabetes (odds ratio (OR)) 1.43, 95\% confidence interval (CI) 1.12 to $1.78 ; \mathrm{p}=0.004$ ) and multivessel disease (OR 1.67, 95\% CI 1.35 to $2.06 ; \mathrm{p}<0.001$ ) were both independent factors related to 10 year mortality. When only the patients with single vessel disease were compared, diabetes was an independent predictor of 10 year mortality, and the impact of diabetes on long term survival became more obvious (OR 1.81, 95\% CI 1.27 to 2.54 ; $\mathrm{p}=0.001$ ) (table 3); on the other hand, diabetes was no longer a predictor of 10 year mortality in patients with multivessel disease (OR $1.17,95 \%$ CI 0.85 to $1.60 ; \mathrm{p}=0.34$ ) (table 4).

\section{Discussion}

Diabetes mellitus has been shown to be a risk factor for the development of coronary artery disease. Epidemiological data from the Framingham study showed there was an increased incidence of coronary heart disease in patients with diabetes mellitus. ${ }^{1}$ The relative risk of myocardial infarction was $50 \%$ greater in diabetic men and $150 \%$ greater in diabetic women. Diabetes is not only a risk factor for the development of myocardial infarction but also a predictor of cardiac mortality after myocardial infarction.

Several studies in the prereperfusion era showed that diabetes has a negative influence on short and long term survival after myocardial infarction. ${ }^{2-4}$ Recent studies have also reported that diabetes is an independent predictor of mortality among patients who underwent reperfusion treatment for acute myocardial infarction. Most of these studies were limited to a one year follow up or less. ${ }^{5-7} \mathrm{~A}$ five year follow up study was reported by Granger and colleagues. ${ }^{8}$ These investigators reviewed the results of the TAMI (thrombolysis and angioplasty in myocardial infarction) study group and showed that diabetes was associated with worse five year survival after infarction. In the current study, we extended the follow up period to 10 years and showed that patients with diabetes had a worse 10 year survival after acute myocardial infarction in the reperfusion era.

It has been reported that diabetic patients have a larger number of risk factors, including older age, more previous infarction, and more severe coronary artery disease. Although the poor prognosis in diabetic patients may in part be attributable to their poor baseline risk profile, most studies have shown that diabetes is an independent predictor of mortality after myocardial infarction. The GUSTO-I (global utilisation of streptokinase and tissue plasminogen activator for occluded coronary arteries) angiographic substudy has reported that diabetes is an independent determinant of 30 day mortality after correction for clinical and angiographic variables. ${ }^{11}$ The current study also showed that diabetes was an independent predictor of 10 year survival using multivariate analysis, adjusting for variables that included multivessel disease. However, it is still possible that concomitant risk factors may affect the relation between diabetes and prognosis after myocardial infarction. Singer and colleagues, comparing a cohort of diabetic patients who had sustained acute myocardial infarction with a similar number of non-diabetic patients, assessed the interaction of diabetes with other preinfarction risk factors. ${ }^{12}$ They reported that the relative risk of 30 day mortality after acute myocardial infarction in diabetic patients was greatest among those with the lowest baseline risk and least among those at highest baseline risk. Because of the lack of angiography in their study, however, the influence of multivessel disease was not determined.

The prognosis of coronary artery disease depends on its severity. Multivessel disease has been shown to be a strong risk factor for mortality after myocardial infarction. Coronary atherosclerosis is not only more prevalent but also more extensive in diabetic than in non-diabetic patients. As previous pathological and angiographic studies have reported, diabetic patients with acute myocardial infarction had a higher incidence of multivessel disease, and both diabetes and multivessel disease were predictors of short and long term mortality. ${ }^{9-12}$ However, the interaction between diabetes and multivessel disease on survival has not yet been studied. In this study, we showed that diabetes was associated with worse long term prognosis after myocardial infarction in patients with single vessel disease. On the other hand, in the presence of multivessel disease, prognosis after myocardial infarction was worsened even in the absence of diabetes, and the influence of diabetes as a risk factor became less obvious.

Diabetic patients are more likely to have multivessel disease and physicians pay a great 
deal of attention to the presence of diabetes in patients with multivessel disease. However, our study showed that diabetes has the greatest impact on long term survival in patients with single vessel disease, not multivessel disease. Thus an aggressive diagnostic and therapeutic approach may be more necessary in patients with acute myocardial infarction and single vessel disease.

There are various possible mechanisms underlying the interaction between diabetes and the vascular involvement in coronary artery disease.

Firstly, diabetic patients with single vessel disease may, in comparison with non-diabetic patients, have more coronary artery atherosclerosis, and this may not be manifest at coronary angiography. Mosseri and colleagues reviewed angiograms of patients with angiographically normal coronary arteries and measured the diameters of their coronary arteries. ${ }^{18}$ They found that the coronary arteries were of smaller diameter in diabetic than in non-diabetic patients, suggesting the presence of diffuse mild atherosclerosis. It has been also reported that diabetic patients are more likely to have moderate stenosis than non-diabetic patients. ${ }^{19}$ Mintz and associates used intravascular ultrasound to evaluate atherosclerosis in angiographically normal coronary artery reference segments and reported that both diabetes and multivessel disease were independent predictors of reference segment per cent cross sectional narrowing. ${ }^{20}$ Mild to moderate atherosclerosis in non-infarct related coronary arteries is thus associated with a poor prognosis in diabetic patients with single vessel disease. As patients with multivessel disease have severe atherosclerosis in non-infarct coronary arteries regardless of whether or not they have diabetes, the influence of diabetes may be diminished in such patients.

Secondly, diabetic patients have a poor baseline risk profile, which in part accounts for their bad prognosis after myocardial infarction. Patients with multivessel disease have so many risk factors that the influence of diabetes may be relatively small. Indeed, in our study, diabetes was associated with more hypertension and hypercholesterolaemia in patients with single vessel disease, while there was no significant difference in these risk factors between diabetic and non-diabetic patients with multivessel disease.

Thirdly, diabetic patients - even in the absence of coronary artery disease-may have abnormalities of systolic and diastolic ventricular function termed diabetic cardiomyopathy. ${ }^{21}$ Interstitial deposits, microvascular disease, and abnormal calcium uptake by the sarcoplastic reticulum are potential causes of diabetic cardiomyopathy. Diabetic cardiomyopathy may contribute to morbidity after myocardial infarction in diabetic patients with single vessel disease whereas ischaemic cardiomyopathy caused by multiple infarctions or hibernation of myocardium may have a greater influence in patients with multivessel disease. ${ }^{22}$
STUDY LIMITATIONS

Our study suffers from the limitations of retrospective analysis. Allocation to reperfusion treatment was based on the physician's decision. However, the type of reperfusion treatment and the incidence of reperfusion were similar between diabetic and non-diabetic patients. A patient was defined as having diabetes if a physician had diagnosed diabetes before or at the time of the current myocardial infarct. Because an oral glucose tolerance test was not performed routinely, some of the diabetic patients may not have been diagnosed as such. However, the incidence of diabetes in our study was comparable with that of previous studies. The diabetic study population had rather mild diabetes on the basis of the treatment they received-only $7 \%$ of them were being treated with insulin. This might have affected the results. It has been reported that insulin treated diabetic patients have a worse outcome after infarction than noninsulin-treated patients. The impact of insulin treated diabetes on survival among patients with multivessel disease might be different from that observed in the current study and this needs to be examined further.

\section{CONCLUSIONS}

Diabetes mellitus is an independent risk factor for long term mortality after acute myocardial infarction in patients with single vessel disease. In patients with multivessel disease, however, the prognosis after myocardial infarction is poor even in the absence of diabetes and the influence of diabetes on long term survival is less obvious.

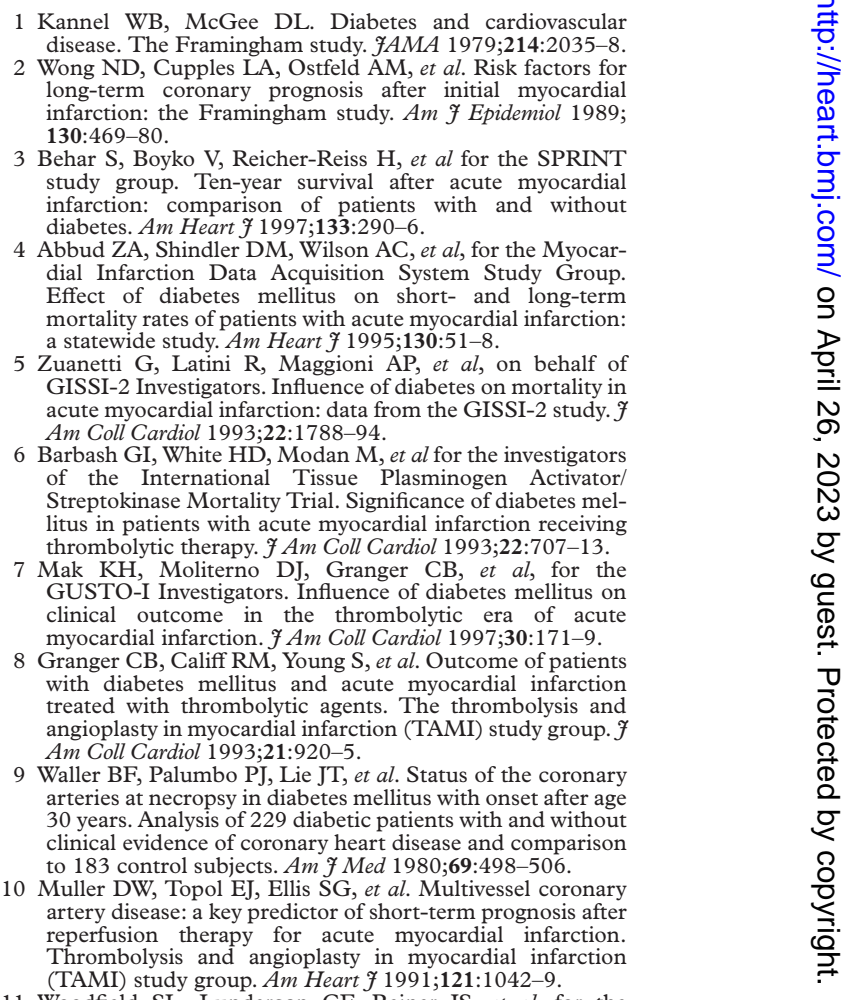

Woodfield SL, Lundergan CF, Reiner JS, et al, for the GUSTO-I angiographic investigators. Angiographic findings and outcomes in diabetic patients treated with thromGUSTO-I experience. $\mathcal{F} \mathrm{Am}$ Coll Cardiol 1996;28:1661-9. 
12 Singer DE, Moulton AW, Nathan DM. Diabetic myocardial infarction: interaction of diabetes with other preinfarction risk factors. Diabetes 1989;38:350-7.

13 LATE study group. Late assessment of thrombolytic efficiency (LATE) study with alteplase 6-24 hours after onset of acute myocardial infarction. Lancet 1993;324:759-66.

14 Cox DA, Rogers WJ, Aguirre FV, et al, and the TIMI Investigators. Effect on outcome of the presence or absence of chest pain at initiation of recombinant tissue plasminogen activator therapy in acute myocardial infarction. $\mathrm{Am} \mathcal{F} \mathrm{Car}$ diol 1994;73:729-36.

15 Ishihara $\mathrm{M}$, Sato $\mathrm{H}$, Tateishi $\mathrm{H}$, et al. Implications of prodromal angina pectoris in anterior wall acute myocardial infarction: acute angiographic findings and long-term prognosis. f Am Coll Cardiol 1997;30:970-5.

16 The TIMI study group. The thrombolysis in myocardial Infarction (TIMI) trial. N Engl f Med 1985;312:932-6.

17 Rentrop KP, Cohen M, Blanke $\mathrm{H}$, et al. Changes in collateral channel filling immediately after controlled coronary artery occlusion by an angioplasty balloon in human subjects. 7 Am Coll Cardiol 1985;5:587-92.

18 Mosseri M, Nahir M, Rozenman Y, et al. Diffuse narrowing of coronary arteries in diabetic patients: the earliest phase of coronary artery disease. Cardiology 1998;89:103-10.

19 Henry P, Makowski S, Richard P, et al. Increased incidence of moderate stenosis among patients with diabetes: substrate for myocardial infarction? Am Heart f 1997;134:

20 Mintz GS, Painter JA, Pichard AD, et al. Atherosclerosis in angiographically "normal" coronary artery reference segments: an intravascular ultrasound study with clinical correlations. F Am Coll Cardiol 1995;25:1479-85.

21 Zarich SW, Nesto RW. Diabetic cardiomyopathy. Am Heart F 1989;118:1000-12.

22 Anversa P, Sonnenblick EH. Ischemic cardiomyopathy: pathophysiologic mechanisms. Prog Cardiovasc Dis 1990; 33:49-70.

\section{IMAGES IN CARDIOLOGY}

\section{Congenitally unguarded tricuspid orifice associated with underdeveloped pulmonary valve leaflets}

This is a transoesophageal echocardiogram of a 23 year old woman who presented with atrial tachycardia and an indefinite past history remarkable for two episodes of anasarka oedema which responded to albumin supplementation. In previous years the patient had exhibited mild to moderate dyspnoea on exertion (New York Heart Association I-II) and palpitations. Physical examination revealed a supine blood pressure of $100 / 75 \mathrm{~mm} \mathrm{Hg}$ and

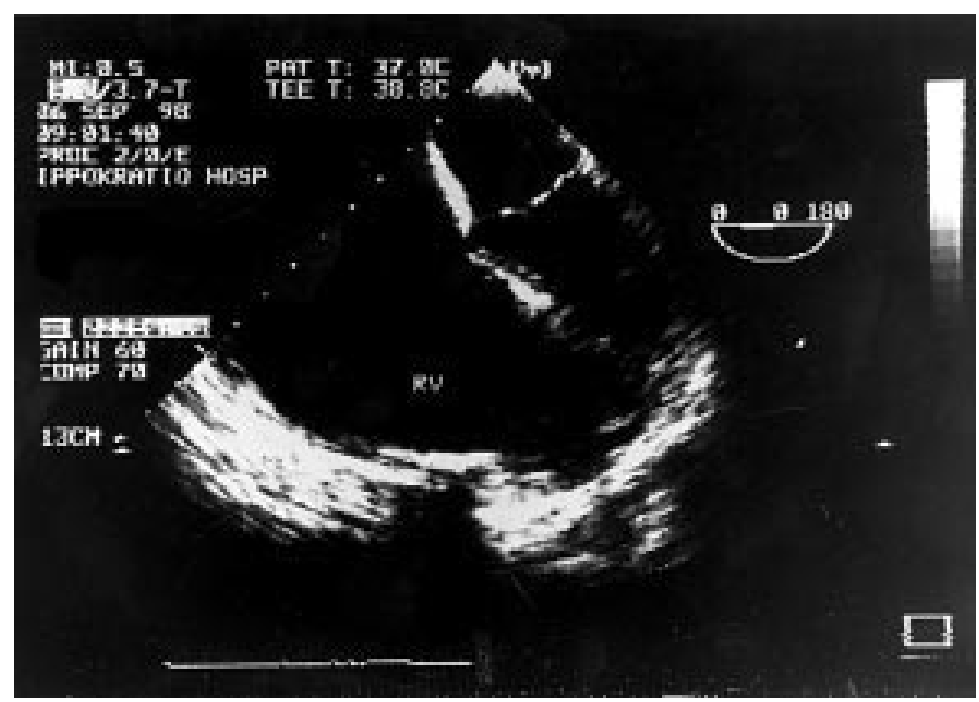

normal S1 and S2. The ECG after cardioversion showed inverted $\mathrm{T}$ waves in precordial leads and a deep and wide $\mathrm{P}$ wave in lead V1. The signal averaged ECG was normal. Transthoracic and transoesophageal echocardiographic study showed that the patient had normal left heart chambers with severely dilated right chambers, absence of tricuspid and pulmonary valve leaflets, with continuous forward pulmonary flow like in Fontan. On cardiac magnetic resonance imaging no high intensity areas, indicating fatty infiltration of the myocardium, were observed. Cardiac catheterisation showed that the right atrium (RA), right ventricle (RV) and pulmonary artery (PA) appeared as a continuous chamber. No tricuspid or pulmonary valve leaflets were detected and the flow was stagnant. The pressure waveform was similar in the RA, RV, and PA trunk with a pressure value of $26 / 12 \mathrm{~mm} \mathrm{Hg}$.

This is an unusual case of a congenitally unguarded tricuspid orifice associated with a patent right ventricular outflow tract and severely underdeveloped pulmonary valve leaflets, mimicking the Fontan circulation. Previous reports have shown that a congenitally unguarded tricuspid orifice is associated with pulmonary atresia.

STELLA BRILI CHRISTODOULOS STEFANADIS PAVLOS TOUTOUZAS stlbrili@ath.forthnet.gr 